\title{
Optimal perioperative anesthesia management for gynecologic interstitial brachytherapy
}

\author{
Alison A. Nielsen, MD', Tehani A. Liyanage, MD', Gary S. Leiserowitz, MD², Jyoti Mayadev, MD³ \\ 'Department of Anesthesiology and Pain Medicine, University of California, Davis Medical Center, Sacramento, CA, ${ }^{2}$ Department of Radiation \\ Oncology, University of California, Davis Medical Center, Comprehensive Cancer Center, Sacramento, CA, ${ }^{3}$ Department of Obstetrics and \\ Gynecology, University of California, Davis Medical Center, Sacramento, CA, USA
}

\begin{abstract}
Purpose: To propose an optimal perioperative pain management clinical care pathway for interstitial brachytherapy for gynecologic cancer based on our interdepartmental experience.

Material and methods: We conducted a retrospective review of 23 women who underwent 32 interstitial brachytherapy procedures for gynecological cancers, analyzing patient demographics, type of anesthetic, medications, postoperative pain scores, adverse events, and delays in discharge. We measured the association of postoperative nausea and/or vomiting (PONV) with hydromorphone use, and postoperative pain scores and total narcotic administration with type of anesthesia.

Results: In 91\% of patients postoperative pain was managed with an epidural infusion plus, as needed (PRN), IV or patient controlled analgesia (PCA) narcotics. The most common postoperative adverse event was PONV (53\%), followed by delirium (22\%). Hospital discharge was delayed, at least by one night, in $26 \%$ of patients. Use of a basal rate on the PCA was associated with all cases of delayed discharge from over-sedation and PONV. The use of $5 \mathrm{mg}$ or more of intravenous (IV) hydromorphone during the first 24-hours postoperatively was associated with PONV $(p=0.01)$. Use of a basal PCA was associated with delirium $(p=0.03)$. Postoperative pain scores were not significantly associated with the type of anesthesia.

Conclusions: Interstitial gynecologic brachytherapy requires a multidisciplinary effort for optimal perioperative management. Our study outlines the appropriate preoperative, intraoperative, and postoperative anesthesia clinical care pathway. Decreased narcotic use during hospitalization and utilization of a patient-directed infusion may decrease side effects and allow for a more efficient hospital discharge.

J Contemp Brachytherapy 2017; 9, 3: 216-223 DOI: https://doi.org/10.5114/jcb.2017.68767
\end{abstract}

Key words: anesthesia, gynecology oncology, interstitial brachytherapy, perioperative.

\section{Purpose}

Interstitial gynecologic brachytherapy involves the placement of a plastic or metal applicator within the target tissue, followed by post-procedural computer controlled automatic after-loading of a radioactive source to allow for high dose radiation to a tumor with better target volume coverage $[1,2,3,4]$. The indications include cervical cancer, in which tumor extension cannot be treated by standard intracavitary brachytherapy alone, endometrial cancer, vaginal cancer, recurrent gynecological cancers or co-morbidities that preclude surgical resection $[1,5,6,7]$. Integrated into the procedure and perioperative course is a multidisciplinary workflow heavily dependent on anesthesia care. Although there is literature addressing anesthesia for brachytherapy procedures, such as for prostate or breast cancer, few studies focus on the optimal anesthe- sia for gynecologic interstitial brachytherapy to help guide brachytherapy best practices and clinicians.

\section{Multidisciplinary team involvement}

The Department of Anesthesiology and Pain Medicine at the University of California, Davis Medical Center, provides anesthesia services for many procedures conducted at the University of California, Davis Comprehensive Cancer Center. The location is not certified for general anesthesia, so monitored anesthesia care (MAC), including deep sedation or neuraxial anesthesia (e.g. spinal, epidural, or combined spinal and epidural anaesthesia [CSE]) is employed. Patients have their interstitial brachytherapy implant placed in the radiation oncology department by a radiation oncologist specializing in gynecological brachytherapy, and are then admitted to the hospital overnight between radiation treatments and

\footnotetext{
Address for correspondence: Dr. Jyoti Mayadev, MD, Assistant Professor, Radiation Oncology Department, Received: 08.09.2016 University of California, Davis Medical Center, 4501 X Street, G140, Sacramento, CA 95817, USA, 
co-managed by the gynecology and radiation oncology services.

\section{Summary of the implant procedure}

Prior to the procedure, most patients are treated with external beam radiation therapy with or without chemotherapy. The radiation oncologist determines the optimal location for interstitial implantation by defining the clinical tumor target and areas at high risk. All patients are then sent to the preoperative anesthesia clinic for evaluation and are scheduled for the procedure. For the procedure, the anesthesiologist places a neuraxial block or provides MAC depending on the needs of the case after discussion with the radiation oncologist. After the patient is in lithotomy position, the cervix or vagina is palpated and a custom vaginal cylinder using a plastic applicator or a plastic applicator supplemented with moldable and adhesive dental putty is placed into the vagina. Thereafter, an external template is placed on the perineum to secure immobilization of the apparatus. Visualization and guidance during the implantation are processed initially by clinical examination. Then, this is aided by real-time transvaginal ultrasound if clinically indicated prior to the insertion of the obturator into the vagina. After the vaginal obturator and template are placed, transrectal and transabdominal ultrasound are performed in tandem or alternating during the procedure. A small thickness computed tomography (CT) is used during the procedure in a limited fashion if 3D imaging is required. Using this template based approach, straight titanium needles are inserted into the perineum and/or vagina, and/or cervix, depending on the target volume and implant technique. The apparatus is secured to the vulva with stitches as shown in Figure 1.

A CT scan is then used to perform better adjustment of the needle placement within the tumor target. Contrast is filled into the bladder ( $25 \mathrm{cc}$ of sterile saline and $5 \mathrm{cc}$ of iodinated contrast) and rectum (15 cc of barium), and a simulation scan is performed for treatment planning purposes. The implantation procedure, CT simulation scan, and needle verification length check takes 2 to 3 hours to complete. The patient is then placed in the nursing holding bay while the treatment planning process (encompassing the contouring portion, needle simulation, physician-generated treatment plan, and physics quality assurance checks), occurs. This process takes 3 to 4 hours, after which the patient is brought back for radiation treatment.

After the procedure, the template and hardware are temporarily left in place, and the patient is admitted to the hospital overnight for 1 to 2 additional high dose radiation treatments the next day, spaced at least 6 hours apart. Because of the retained hardware, it is crucial to provide continued analgesia overnight. Often a continuous epidural, with or without systemic analgesics are needed. After the last treatment, the applicators are removed and analgesic requirements are minimal. The epidural is removed and once the patient is stable, she is being discharged home.

\section{Material and methods}

With an intent to develop an optimized perioperative care pathway, and after approval from our Institutional
Review Board (IRB), we conducted a retrospective review of anesthesia and hospital records for all patients who underwent an interstitial gynecologic brachytherapy procedure over a 4-year period from 2010-2013 at the University of California, Davis Comprehensive Cancer Center in Sacramento, California. Data was collected by a single researcher through review of patients' electronic medical records and scanned written anesthesia records for patient demographics, baseline laboratory values, type of anesthesia, medication administration, patient vitals, pain scores, and side effects. Data are presented as summary statistics (using means, medians, ranges, interquartile range, and proportions).

We cataloged the following complications: postoperative nausea and/or vomiting (PONV), respiratory depression (defined as use of naloxone or arousal difficulty with need for supplemental oxygen), delirium (as noted by physician progress notes), urinary retention (inability to void after Foley removal), postoperative hypotension (blood pressure $<20 \%$ of preoperative value), and delayed discharge (at least one unplanned overnight stay). We examined the mean of all the pain scored recorded. Generally, pain scores are recorded every 6 hours or during a transition of care. We calculated a mean for all postoperative pain scale data available.

Postoperative narcotic administration was measured in the form of IV administered hydromorphone. In instances where oral narcotics or IV morphine were given, an appropriate opioid conversion was used to convert to IV hydromorphone equivalents, using the Global RPh opioid conversion calculator (http://www.globalrph. com/narcoticonv.htm).

\section{Statistical analysis}

The Fisher exact probability test was employed for statistical analysis to investigate the relationship between postoperative delirium and patient controlled analgesia (PCA) use, delirium and scheduled promethazine or lorazepam combined with a narcotic epidural, and PONV and IV hydromorphone administration. Significance was held at the standard value of $p<0.05$. A Kruskal-Wallis test was used to compare postoperative narcotic admini-

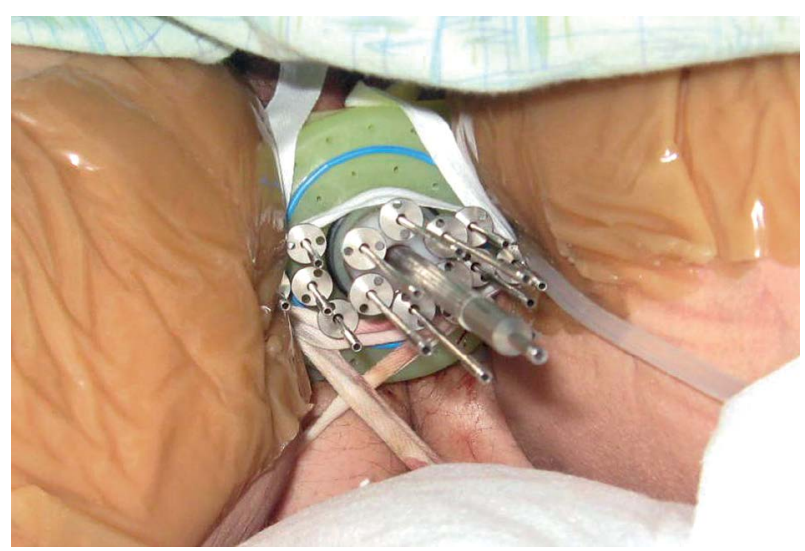

Fig. 1. Implant device with retained hardware for additional spaced radiation treatments 
stration between MAC/local anesthetics, epidural, and combined spinal epidural (CSE). Statistical analysis was done using Vassar Stats platform for statistical computation. Whisker box plots were generated using QI Macros - six sigma for Excel platform.

\section{Results}

\section{Patient demographics}

During the study period, 27 patients underwent interstitial brachytherapy for gynecologic cancers. Four were excluded from the study because they did not have perineal interstitial brachytherapy implantation. For the remaining 23 patients included, 32 implant procedures were performed on separate occasions and reviewed. One patient was not admitted for multiple treatments as an inpatient, but was monitored in a prolonged recovery setting; therefore only limited data was available and analyzed. All patients were women with advanced or recurrent gynecological cancers of the cervix, vagina, or endometrium who received high dose rate radiation treatments. The mean patient age was 55 years with a range of 31-84 years. The American Society of Anesthesiologist (ASA) classification is a system for assessing the fitness of patients before surgery: 1 - healthy person; 2 - mild systemic disease; 3 - severe systemic disease; 4 - severe systemic disease that is a constant threat to life; 5 - moribund person who is not expected to survive without the operation; 6 - declared brain dead person who's organs are being removed for donor purposes. Seventy-four percent of patients were classified as ASA physical class III or IV. The mean body mass index (BMI) was $28 \mathrm{~kg} / \mathrm{m}^{2}$ with a range of $17-51 \mathrm{~kg} / \mathrm{m}^{2}$. The patients' laboratory results are shown in Table 1 . The most common electrolyte abnormalities included hypokalemia

Table 1. Distribution of age, the American Society of Anesthesiologist physical classification, weight, and laboratory findings of patients undergoing interstitial brachytherapy for gynecological cancer

\begin{tabular}{lc}
\hline Patient characteristic & Value \\
\hline Age (years), mean (range) & $55(31-84)$ \\
\hline ASA physical status 2 & $26 \%(6 / 23)$ \\
\hline ASA physical status 3 & $70 \%(3 / 23)$ \\
\hline ASA physical status 4 & $4 \%(1 / 23)$ \\
\hline BMI (kg/m²) mean (range) & $28(17-51)$ \\
\hline Underweight (BMI < 18.5) & $4 \%(1 / 23)$ \\
\hline Normal (BMI 18.5-24.9) & $30 \%(7 / 23)$ \\
\hline Overweight-severe (BMI 25-39.9) & $57 \%(13 / 23)$ \\
\hline Morbid obesity (BMI $\geq 40)$ & $9 \%(2 / 23)$ \\
\hline Hemoglobin 10-12 (g/dl) & $50 \%(8 / 16)$ \\
\hline Hemoglobin 8-10 (g/dl) & $50 \%(8 / 16)$
\end{tabular}

ASA - American Society of Anesthesiologist, BMI - body mass index
( $\mathrm{K}<3.3 \mathrm{mEq} / \mathrm{l})$ in $50 \%$ of patients tested, hypomagnesemia $(\mathrm{Mg}<1.5 \mathrm{mEq} / \mathrm{l})$ in $86 \%$ of patients, and elevated creatinine $(\mathrm{Cr}>1.27 \mathrm{mg} / \mathrm{dl})$ in $19 \%$ of patients, resulting from chemoradiation or the cancer disease process, as shown in Table 1.

\section{Anesthetic technique}

The type of anesthesia used is determined by attending anesthesiologist and radiation oncologist. Typically, our institution uses an epidural, so that the pain control is continuous in the hospital setting overnight.

In the absence of a general anesthetic, patients need adequate reassurance and sedation to tolerate the procedure, even if analgesia is being provided by an epidural or combined spinal and epidural (CSE), as the patient is standardly placed into the lithotomy position for many hours. It is important to properly position the legs and arms to avoid nerve damage. Sedation can be provided by continuous or intermittent propofol, or sporadic midazolam as needed. Applicators that are inserted into the uterus and vagina, often require a T10-L1 blockade. The bladder is often distended for imaging purposes, which requires at least a T10 blockade and may cause abdominal cramping despite neuraxial anesthesia. Insertion of a rectal ultrasound probe or applicator usually requires sacral blockade. Distension of the cervix, upper vaginal packing, and labial sutures are often carried by the pudendal S2-S4 nerve roots [8]. Spinal anesthesia may improve sacral blockade, while an epidural can be used for continuous analgesia intraoperatively and postoperatively for subsequent radiation series.

An epidural was the most common anesthetic used for the procedure $(53 \%)$, followed by combined spinal epidural $(34 \%)$, and MAC with paracervical block (13\%). If a spinal epidural was used, $0.75 \%$ bupivacaine at $1-1.5 \mathrm{ml}$ was the most commonly used. For epidural dosing during the implant procedure, $0.25 \%$ bupivacaine divided to a total volume of $10-15 \mathrm{ml}$ was the most commonly used. Intrathecal (aka spinal) or epidural narcotics were used in $43 \%$ of the neuraxial anesthetics, with single dose fentan$\mathrm{yl}$ added in $50 \%$ of these cases, while the other $50 \%$ had a continuous fentanyl or hydromorphone epidural with local infusion during and/or after the procedure. Epidural infusions were started during the implant procedure and continued until implant removal. Most of the local or local/ narcotic epidural infusions were run between $8-12 \mathrm{ml} / \mathrm{hr}$.

The most commonly used intraoperative IV medications were midazolam and fentanyl. Patients received a mean of $4 \mathrm{mg}$ of midazolam and $100 \mu \mathrm{g}$ of fentanyl. In one-fourth of cases, low-dose propofol infusion $(<100 \mu \mathrm{g} /$ $\mathrm{kg} / \mathrm{min}$ ) or intermittent boluses was used. Less commonly used IV adjuncts included: dexmedetomidine (either as a bolus or drip, with a total $<0.5 \mu \mathrm{g} / \mathrm{kg}$ ), ketamine (10-25 mg), or remifentanil (drip range of $0.03-0.05 \mu \mathrm{g} / \mathrm{kg} /$ $\mathrm{min}$ ). The most commonly administered intraoperative antiemetic was IV ondansetron (4 mg).

\section{Postoperative management}

Of the 31 implant procedures requiring hospital admission, $28(90 \%)$ had a continuous postoperative epi- 
dural (most commonly $0.1 \%$ bupivacaine at $8-12 \mathrm{ml} / \mathrm{hr}$ ), with $6(21 \%)$ having fentanyl $(2 \mu \mathrm{g} / \mathrm{ml})$, or hydromorphone $(0.02 \mathrm{mg} / \mathrm{ml})$ added to the epidural local infusion. The most common postoperative IV analgesic administered was hydromorphone. Of the 31 patients, 19 (61\%) had PRN IV hydromorphone or PCA without a basal rate. No patients were given a basal rate on their PCA if they had a continuous narcotic in their epidural infusion. In addition, 11 patients (35\%) were given oral hydrocodone or oxycodone with $325 \mathrm{mg}$ of acetaminophen during the hospitalization, with a mean of $17.5 \mathrm{mg}$ of oral narcotic over the entire hospital stay. Acetaminophen, in intravenous form, was only given to 2 patients and ibuprofen was used in 1 patient. Antiemetics were given on an as-needed basis or scheduled. Promethazine was the most commonly administered, followed by metoclopramide and ondansetron. Postoperative anxiolytics were administered to 16 patients (52\%). The most commonly used anxiolytic was lorazepam (10 patients, 63\%), followed by zolpidem (3 patients, 19\%), haloperidol (2 patients, $12 \%$ ), and alprazolam (1 patient, $6 \%$ ).

\section{Pain scores and postoperative narcotic administration}

Mean postoperative pain scores, by use of the visual analog scale (0 meaning "no pain" and 10 meaning "worst imaginable pain"), were calculated for each anesthetic type. The mean pain score was 3.0, 3.3, and 2.6 in the MAC/local, epidural, and CSE, respectively. However, one MAC/local patient had very low scores recorded (0-1), but received a high amount of narcotics. On review of the records, she did not speak English. Another patient in the MAC/local group stayed less than 24 hours and had no postoperative pain scores recorded. A Kruskal-Wallis test was used to analyze differences in postoperative pain scores of the three groups, which were not found to be statistically significant $(p=0.22)$.

After the initial implant procedure, the median amount of equivalent IV hydromorphone administered over 24 hours was $4.8 \mathrm{mg}$ with epidural (Q1-Q3: 2.25-8.5 mg),

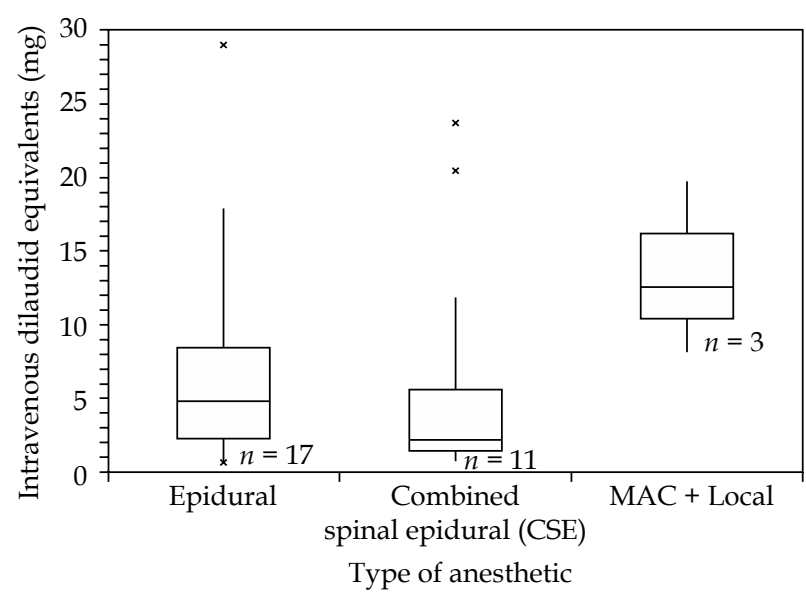

Fig. 2. Postoperative narcotic administration by type of primary anesthetic using a box and whiskers plot
$2.2 \mathrm{mg}$ with CSE (Q1-Q3: 1.45-5.6 mg), and $12.6 \mathrm{mg}$ with MAC/local (Q1-Q3: 10.4-16.2 mg). A Kruskal-Wallis test was used to compare the three groups. Use of a CSE was associated with 2.2-fold decrease in the total narcotic administration compared to epidural, and a 5.7-fold decrease in narcotic administration compared to MAC/local, as shown in Figure 2, but these differences were not found to be statistically significant $(p=0.10)$.

\section{Side effects, complications, delayed discharges}

PONV was present in 17 patients (53\%). Of these, $35 \%$ had narcotics in their neuraxial anesthetic, while $65 \%$ did not. There was a statistically significant difference in the incidence of PONV with the use of $5 \mathrm{mg}$ or more of IV hydromorphone equivalents in the first 24 hours of postoperative course compared to less than $5 \mathrm{mg}(p=0.01)$, as shown in Figure 3. Agitation, delirium, or oversedation was noted in 7 patients (22\%), of these $5(71 \%)$ were given a basal rate on their PCA. The association of delirium with basal PCA use was found to be statistically significant using the Fisher exact probability test $(p=0.03)$.

Respiratory depression occurred after 2 of the 32 procedures $(6 \%)$. In one case, the patient received scheduled promethazine and lorazepam with epidural narcotics and a hydromorphone PCA. In the other case, the patient was receiving scheduled promethazine with epidural narcotics and a hydromorphone PCA. However, the use of scheduled promethazine and/or lorazepam plus narcotic epidural was not found to be significantly associated with respiratory depression $(p=0.11)$. Foley catheters were typically removed 6-8 hours after the epidural was stopped. Urinary retention occurred in 1 of 32 procedures, requiring re-insertion of the Foley catheter. Postoperative hypotension (blood pressure $<20 \%$ baseline) occurred in 4 patients (13\%), in 3 who had epidurals, and in one who had a CSE, as summarized in Table 2.

Of 32 brachytherapy procedures, 7 sessions (22\%) required a delay in the hospital discharge requiring an overnight stay due to blood transfusion, $(3 / 7,43 \%)$, fever/

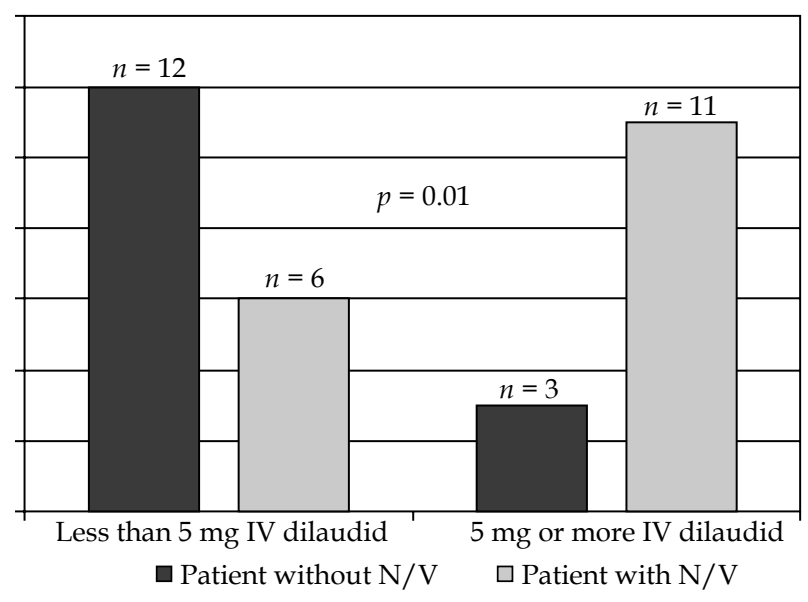

Fig. 3. Postoperative narcotic administration (dilaudid equivalents) over a 24-hour period and incidence of postoperative nausea and/or vomiting (N/V) 
Table 2. Distribution of postoperative adverse events or side effects

\begin{tabular}{lc} 
Nausea and/or vomiting & $53 \%(17 / 32)$ \\
\hline Agitation, delirium, over-sedation & $22 \%(7 / 32)$ \\
\hline Postoperative hypotension & $13 \%(4 / 32)$ \\
\hline Respiratory depression & $6 \%(2 / 32)$ \\
\hline Urinary retention & $3 \%(1 / 32)$
\end{tabular}

sepsis/additional work-up $(2 / 7,28 \%)$, electrolyte abnormality $(1 / 7,14 \%)$, and social issues $(1 / 7,14 \%)$.

\section{Discussion}

There is paucity in the literature describing the anesthesia workflow and analgesic requirements for each step of interstitial gynecologic brachytherapy. Jain et al. compared different anesthetic techniques for intracavitary radiotherapy for cervical cancer, finding that neuraxial anesthesia provided better postoperative analgesia with fewer side effects as compared to general anesthesia [9]. In a retrospective analysis, by Benrath et al. neuraxial anesthesia was the predominant technique for pelvic brachytherapy [10]. Epidural analgesia was found to provide satisfactory pain relief for pelvic brachytherapy in a study by Janaki et al. [8]. However, none of these series examined the perioperative anesthetic requirements for interstitial brachytherapy. Intracavitary brachytherapy only requires applicators to be inserted for several hours, while interstitial implants require perineum, vaginal sutures, and hardware retained in the patient overnight for a series or radiation treatments, thus necessitating optimal anesthesia care. We sought to further examine our perioperative anesthesia care to improve our patient experience, and investigate quality control metrics, such as prolonged hospitalization rates.

\section{Perioperative considerations and challenges}

There are several important considerations and challenges for this particular patient population and procedure. We summarize observations from our patient series, which guided our development of a proposed perioperative care pathway, and which we have divided into sections relative to the procedure.

\section{Preoperative}

Patients undergoing interstitial brachytherapy are often in a higher risk category for anesthesia complications given their co-morbidities. In our study, $74 \%$ of patients were classified as an ASA III or IV. Patients may have electrolyte abnormalities from gastrointestinal disturbances, volume depletion, chemotherapy, and external beam radiation treatments. In our study, hypokalemia and hypomagnesemia were the most common electrolyte abnormalities. In our preoperative clinic, a laboratory workup is often ordered, but results may not be acted upon until the day of the procedure.
Ideally, preoperative screening and correction of any severe electrolyte abnormalities should occur before the procedure.

Significant symptomatic anemia or thrombocytopenia may complicate the anesthetic technique or delay discharge. In our study, 4 of 7 delays in hospital discharge involved blood transfusion for symptomatic anemia. Thrombocytopenia was present in $40 \%$ of patients tested. In addition, patients may be on systemic anticoagulants for thromboembolic events associated with their malignancy, which may preclude use of a neuraxial anesthetic. We usually hold the procedure if the patient's platelet count is less than $70 \mathrm{~K} / \mathrm{mm}^{3}$ and consider platelet transfusion. We suggest that all patients should be screened for symptomatic anemia. A preoperative platelet count should be considered for neuraxial anesthesia. Patient medications and history should be carefully reviewed before placement of a neuraxial block.

\section{Intraoperative}

A variety of anesthetic techniques for pelvic brachytherapy have been reported, including general anesthesia, epidural, CSE, and MAC with a pudendal or paracervical block $[6,8,9,10,11,12]$. In our study, the most common anesthetic technique was an epidural, followed by a CSE. We did not perform any procedures under general anesthesia because of certification restrictions in the cancer center.

The implant procedure is often performed in ancillary locations, which may preclude certain equipment or general anesthesia. At our institution, the procedure is conducted in an outpatient ancillary setting, where there is access to a radiation simulator, and high-dose-rate treatment. Intraoperative challenges for the anesthesia provider include restricted airway access because of limitations in the ancillary suite, patient positioning, darkness of the room for ultrasound use, and monitor incompatibility with imaging devices.

\section{Postoperative}

After the procedure, patients are admitted for inpatient monitoring. They retain the applicators for radiation afterloading the next day. Patients are kept on strict bed rest with continued analgesic requirements, which may be managed with a continuous epidural (with or without narcotics) and/or systemic opioids and adjunctive pain medications. In our study, the use of a CSE was associated with a 2.2-fold reduction in total postoperative narcotic administration compared to an epidural, and a 5.7-fold reduction in narcotic use compared to MAC/local anesthetic. The association of less narcotic administration with use of a CSE may be explained by a spinal providing a denser block with greater sacral spread, such that vaginal hardware was better tolerated, compared to an epidural.

We found that the incidence of PONV significantly increased with use of $5 \mathrm{mg}$ or more of IV hydromorphone in the first 24-hours postoperatively. In order to decrease the overall postoperative narcotic administration, we recommend eliminating the basal rate of the PCA. Patients 
may be receiving more narcotics than needed with a basal rate and suffering increased side effects. Optimizing the epidural infusion and using adjunctive therapies may decrease the overall opioids administered, while still providing adequate pain relief. We suggest scheduling oral or IV acetaminophen throughout the hospitalization to potentially decrease the overall opioid administration. Ibuprofen and ketorolac are often avoided because of their potential to worsen vaginal bleeding.

In our study, a hydromorphone PCA was commonly used with an epidural for postoperative analgesia. We found that use of a basal rate with the PCA was significantly associated with an increase in delirium. Of the 7 patients in this study with documented delirium or oversedation, 5 had a continuous basal PCA and 2 had scheduled promethazine or lorazepam with a narcotic epidural. Because of their immobility, patients may have worsening feelings of anxiety or claustrophobia. Scheduled anxiolytics, such as lorazepam, may pose a risk of increased delirium or respiratory compromise when combined with epidural or IV narcotics. If a patient is anxious, despite reassurance and effective analgesia, we suggest only as-needed anxiolytics. Caution must be taken in prescribing anxiolytics in patients receiving concurrent neuraxial narcotics or basal PCA.

In our study, $53 \%$ of patients had PONV. This is a much higher rate of $\mathrm{N} / \mathrm{V}$ than reported in other brachytherapy studies with non-interstitial patients. Benrath et al. reported PONV in only $1.3 \%$ of their patients. However, only $17 \%$ of their patients had tumors of the female genital organs and all types of brachytherapy procedures were included, even just removal of applicators or single dose therapies [9]. Our study only included patients receiving interstitial brachytherapy implants for gynecological cancers. Our patient population is considered moderatehigh risk for PONV given female sex, use of opioids, and gynecological procedure/manipulation [13]. Because of the retained hardware, the gynecological organs are being manipulated throughout hospitalization, which may contribute to such a high rate of PONV.

Promethazine was the most commonly used postoperative antiemetic in our study, which is considered by the Society for Ambulatory Anesthesia to have only some antiemetic efficacy [14]. Based on our high rates of PONV, we recommend that patients receive prophylactic antiemetics during the initial procedure. Ondansetron is recommended as first-line prophylaxis, and combination therapy (adding another antiemetic in a different class) is recommended in moderate-high risk patients to increase effectiveness [14]. Antiemetics with sedating properties (e.g. promethazine), should be used with caution especially in the elderly patients, as they may promote delirium or have synergistic respiratory depressive effects when combined with opioids.

Non-pharmacological interventions to reduce PONV include maintaining appropriate hydration status. Oral intake may be difficult because of PONV, immobility, and limited time for intake between subsequent radiation procedures; therefore, hydration status should be frequently assessed and maintenance fluids given. If the patient can tolerate fluids orally, consider giving fluids as soon as can be tolerated after the procedure.

While in the hospital, after we discontinue the epidural, the patient is offered oral pain medication as needed. They are then discharged according to their preoperative outpatient pain regimen and follow closely with radiation oncology for pain medication management.

\section{Discharge readiness}

Analgesia is needed for repeated brachytherapy fractions during hospital admission, but is usually tolerated with minimal IV sedation or with a continuous epidural infusion, as the applicators and hardware are already in place. After the last radiation treatment, the applicators are removed, and often the patient is given short term intravenous or oral medications due to pain with the applicator removal. The epidural catheter is then removed at this time. The patient must be assessed for readiness for discharge. In our study, discharge was delayed because of abnormal electrolytes, symptomatic anemia, fever/sepsis/additional work-up $(2 / 7,28 \%)$, and social issues $(1 / 7,14 \%)$. To reduce discharge delays, patients should be screened early preoperatively and postoperatively for symptomatic anemia, and electrolytes abnormalities should be addressed, ideally starting in the preoperative clinic. Oversedation or delirium may be reduced with general delirium prophylaxis, minimization of IV narcotics, and avoidance of scheduled anxiolytics or sedating antiemetics.

\section{Proposed perioperative pathway}

Perioperative clinical pathways are structured multidisciplinary care plans that detail the essential steps in the care of patients with specific clinical problems or interventions, with a goal to improve the overall process and outcome. Pathways have been found in numerous studies to reduce costs, decrease variation in patient care, and improve quality and satisfaction of patient care [15,16,17].

Each step in the process of interstitial gynecologic brachytherapy requires meticulous quality assurance. Analysis of workflow facilitates the exploration of needed efficiency within each designated task for potential feedback and troubleshooting. After evaluation of our institutional experience, we designed an interdisciplinary perioperative pathway for patients receiving interstitial brachytherapy for gynecologic cancers, summarized as a flowchart in Figure 4.

\section{Implementing a perioperative pathway}

Our perioperative pathway could be adopted or adapted by hospital interdepartmental clinical care committees to develop a standard regimen for patients undergoing interstitial brachytherapy for gynecological cancers. Other details or components could be added, for example, subcutaneous heparin schedules for deep vein thrombosis prophylaxis, bowel prep regimens, NPO status, prophylactic antibiotic dose/times, use of Lomotil (diphenoxylate and atropine) to reduce bowel movements, timing of ambulation, nursing care plans, and discharge criteria. Scheduling details, preoperative clinic timing, procedural 


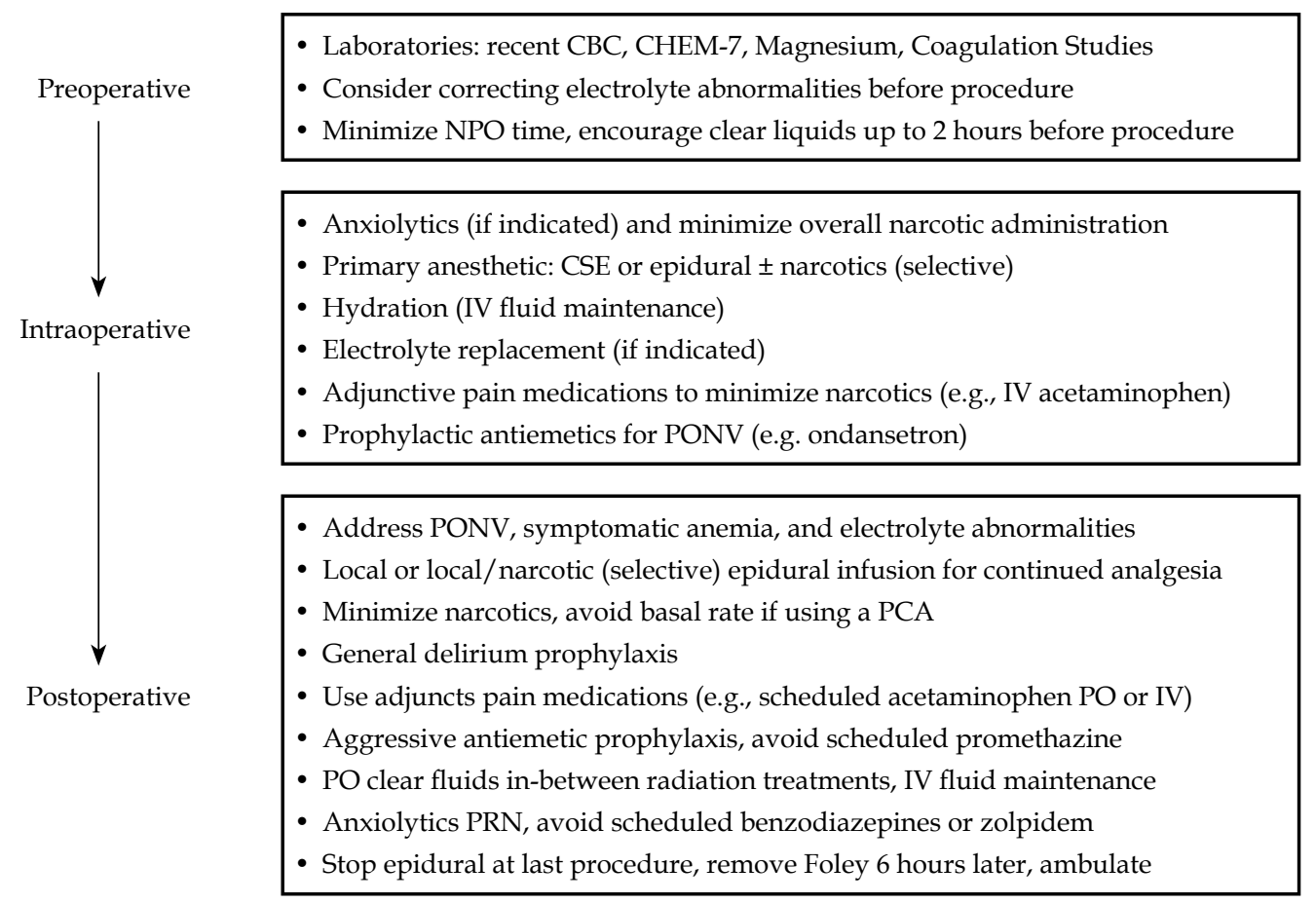

Fig. 4. Proposed perioperative pathway for interstitial brachytherapy for gynecological cancers, describing important considerations for the preoperative, intraoperative, and postoperative periods

scheduling, and timing for additional radiation therapy could also be added. Patient outcomes, patient satisfaction, hospital costs, and side effects should be tracked over time and the pathway modified as needed.

\section{Limitations and future research}

A limitation of our study is our small sample size and different combinations of medications that were used. However, all epidural and spinal anesthesia were administered at as a combination and rate according to our anesthesia departmental standards. Although the specific combination would vary based on the patient history and anticipated pain, we wanted to exemplify the use of the spinal and epidural anesthesia in perioperative pain management for gynecologic interstitial brachytherapy. We did use an opiate conversion calculator to convert all IV postoperative pain medications into a common drug for comparison.

Additionally, we did not collect data regarding patient reported satisfaction with the procedure and anesthesia; rather, we used VAS scores and narcotic administration as a proxy for the degree of analgesic satisfaction. Ideally, a future study would specifically interview for patient satisfaction with the procedure and postoperative period. Because this was a retrospective study, patients were not randomized to receive specific types of anesthesia, so there may be selection bias of which patients received what type of anesthesia and postoperative analgesia. We did not perform any procedures under general anesthesia, so we were unable to compare general to neuraxial anesthetic techniques.

Another limitation of our study is that the number of interstitial needles or location of the target volume could have influenced the pain score of the patient in the perioperative period. For example, we suspect the response to preoperative external beam radiation would influence the number of catheters placed. Although not shown currently in the literature, a higher number of catheters may correlate with an increase in the pain scale, which could be a confounder in our dataset.

Our data is increasingly important, as hospitals and outpatient based clinics examine efficiency and discharge delays as a quality metric. In the near future, quality metrics will be linked to physician and hospital reimbursements and national quality scores. We plan to implement our perioperative pathway through our institution's clinical care committee and compare patient outcomes and workflow efficiency in future studies.

\section{Acknowledgements}

The authors would like to thank Dr. Aimee Sisson and Dr. Neal Fleming for their careful review and editing of the manuscript.

\section{Disclosure}

Authors report no conflict of interest.

\section{References}

1. Beriwal S, Demanes DJ, Erickson B et al. American Brachytherapy Society consensus guidelines for interstitial brachytherapy for vaginal cancer. Brachytherapy 2012; 11: 68-75.

2. Nag S, Erickson B, Parkh S et al. The American brachytherapy society recommendations for high-dose-rate brachytherapy for carcinoma of the endometrium. Int J Radiation Oncology Biol Phys 2000; 48: 779-790. 
3. Aridgides $\mathrm{P}$, Onderdonk B, Cunningham $\mathrm{M}$ et al. Institutional experience using interstitial brachytherapy for the treatment of primary and recurrent pelvic malignancies. J Contemp Brachytherapy 2016; 8: 173-180.

4. Murakami N, Kobayashi K, Kato T et al. The role of interstitial brachytherapy in the management of primary radiation therapy for uterine cervical cancer. J Contemp Brachytherapy 2016; 8: 391-398.

5. Erickson B, Gillin M. Interstitial implantation of gynecologic malignancies. J Surg Oncol 1997; 66: 285-295.

6. Ash D. Interstitial Brachytherapy in Gynecological Cancer. In: The GEC ESTRO handbook of brachytherapy. Leuven (Belgium): ACCO 2002; 17: 417-433. Available from: http:// estro-education.org/publications/Documents/final\%20introduction.pdf. Accessed 20 April 2014.

7. Kanna N, Beriwal S, Kim H et al. High-dose-rate interstitial computed tomography based brachytherapy for the treatment of cervical cancer: Early results. Brachytherapy 2012; 11: 408-412.

8. Janaki MG, Nirmala S, Kadam AR et al. Epidural analgesia during brachytherapy for cervical cancer patients. J Can Res Ther 2008; 4: 60-63.

9. Jain R, Mishra S, Bhatnagar S. Comparison of Different Anaesthetic Techniques for High-dose-rate Intracavitory Radiotherapy for Carcinoma Cervix. The Internet Journal of Anesthesiology 2008; 17: [about 5pp.]. Available from: http://ispub. com/IJA/17/1/11110. Accessed 20 April 2014.

10. Benrath J, Kozek-Langrenecker SK, Hupfl M et al. Anaesthesia for brachytherapy $-51 / 2$ yrs. of experience in 1622 procedures. Br J Anaesth 2006; 96: 195-200.

11. Appadurai IR, Hanna L, Symonds RP. Analgesia for pelvic brachytherapy. B J Anaesth 2002; 89: 342-343.

12. Smith MD, Todd JG, Symonds RP. Analgesia for pelvic brachytherapy. Br J Anaesth 2002; 88: 270-276.

13. Gan TJ. Risk factors for postoperative nausea and vomiting. Anesth Analg 2006; 102: 1884-1898.

14. Gan TJ, Meyer TA, Apfel CC et al. Society for Ambulatory Anesthesia guidelines for management of postoperative nausea and vomiting. Anesth Analg 2007; 105: 1615-1628.

15. Adesanya AO, Joshi GP. Hospitalists and anesthesiologist as perioperative physicians: Are their roles complementary? Proc (Bay Univ Med Cent) 2007; 20: 140-142.

16. Marcario A, Horne M, Goodman S et al. The effect of a perioperative clinical pathway for knee replacement surgery on hospital costs. Anesth Analg 1998; 86: 978-984.

17. Napolitano LM. Standardization of perioperative management: clinical pathways. Surg Clin North AM 2005; 85: 1321-1327. 